\title{
Initial Serum Glucose Level as a Prognostic Factor in the First Acute Myocardial Infarction
}

\author{
Chin-Wang Hsu, MD, MS \\ Hsiu Hsi Chen, MD, PhD \\ Wayne H.-H. Sheu, MD, PHD \\ Shi-Jye Chu, MD \\ Ying-Sheng Shen, MD \\ Chin-Pyng Wu, MD, PHD \\ Kuo-Liong Chien, MD, PhD
}

\begin{abstract}
From the Department of Emergency Medicine, Tri-Service General Hospital, Taipei, Taiwan (Hsu, Chu, Shen, Wu); the Institute of Preventive Medicine, College of Public Health, National Taiwan University, Taipei, Taiwan (Chen, Chien); and the Department of Medical Research and Education, Taichung Veterans General Hospital, Taipei, Taiwan (Sheu).
\end{abstract}

Study objective: We assess the prognostic role of initial glucose levels in patients with a first acute myocardial infarction in the emergency department (ED).

Methods: We conducted a 3-year retrospective cohort study. Patients with a first acute myocardial infarction were recruited from the ED of a tertiary hospital from January 1, 2001, to December 31, 2003. Initial glucose levels in the ED were stratified into 3 levels (normal $<140 \mathrm{mg} / \mathrm{dL}$; intermediate 140 to $200 \mathrm{mg} / \mathrm{dL}$; and high $\geq 200 \mathrm{mg} / \mathrm{dL}$ ). Logistic and Cox regression models were applied to estimate the 1-month short-term and 1-year long-term adverse prognoses, respectively.

Results: A total of 198 eligible subjects (159 men and 39 women; mean age $63.1 \pm 14.2$ years) were recruited. The estimated survival curves among the 3 initial glucose levels were significantly different $(P=.0002)$. After adjustment for sex, age, diabetic status, reperfusion therapy, and infarct subtype, the adjusted odds ratio for short-term prognosis progressed with higher levels when compared with the normal level (intermediate level: odds ratio 3.87; 95\% confidence interval $[\mathrm{Cl}]$ 1.71 to 8.78 ; high level: odds ratio $5.16 ; 95 \% \mathrm{Cl} 1.97$ to 13.51). High initial glucose level was an important risk factor for long-term adverse prognosis (hazard ratio 3.08; $95 \% \mathrm{Cl} 1.59$ to 5.98 ).

Conclusion: A high initial glucose level in the ED is an important and independent predictor of shortand long-term adverse prognoses in patients with first acute myocardial infarction. [Ann Emerg Med. 2007;49:618-626.]

\section{INTRODUCTION}

Acute myocardial infarction is classified as a critical illness in the emergency department (ED). Patients can die suddenly from ventricular arrhythmia or cardiogenic shock. Hyperglycemia of more than $144 \mathrm{mg} / \mathrm{dL}$ is observed in at least $50 \%$ of patients with acute myocardial infarction. ${ }^{1,2}$ There is also a positive association between early hyperglycemia at the event and the prognosis of acute myocardial infarction. Hyperglycemia on admission, irrespective of whether the patient is diabetic, is an independent predictor of cardiac outcome, including congestive heart failure, cardiogenic shock, and death after acute myocardial infarction. ${ }^{3-5}$ Many studies have demonstrated a higher risk of short- and long-term adverse prognoses in patients with hyperglycemia on admission for acute myocardial infarction. ${ }^{6-12}$
However, the association between initial glucose levels at the $\mathrm{ED}$ and prognoses after first acute myocardial infarction has rarely been discussed. The initial glucose level at the ED represents the early glucose response to acute myocardial infarction and will not be influenced by glucose-containing solutions or treatment by the referring institution. Furthermore, short- and long-term adverse prognoses after acute myocardial infarction have not been assessed simultaneously in many studies involving initial glucose levels. The stress factor for the first acute myocardial infarction subjects is susceptible to neurohumoral response and not confounded by the effect of interventions in coronary artery disease or previous acute myocardial infarction patients who may have a reduced stress hormone response to recurrent acute myocardial infarction. The 


\section{Editor's Capsule Summary}

What is already known on this topic

Diabetic patients who sustain acute myocardial infarction have a worse prognosis than nondiabetic patients.

What question this study addressed

This study addressed the relationship between initial glucose level and outcome in patients with acute myocardial infarction.

What this study adds to our knowledge

The long-term outcome of acute myocardial infarction patients with even a mild hyperglycemia (140 to 200 $\mathrm{mg} / \mathrm{dL}$ ) on emergency department arrival is worse than patients without hyperglycemia, regardless of whether they have preexisting diabetes.

How this might change clinical practice

This study should not change practice. Possibly, the initial glucose level is a surrogate for a more profound stress hormone response in more ill patients. Until it is shown that more aggressive treatment of these patients improves outcome, it is simply an interesting observation.

duration of acute myocardial infarction more than 24 hours may not reflect the peak glucose response. Besides, having recurrent myocardial infarction or receiving interventions for severe coronary artery disease may result in fewer or more adverse outcomes of acute myocardial infarction compared with first acute myocardial infarction. ${ }^{13,14}$ This study investigated the association between initial glucose levels at the ED and the short- and long-term cardiac prognoses in patients with first acute myocardial infarction.

\section{MATERIALS AND METHODS}

\section{Study Design and Selection of Participants}

This is a 3-year retrospective cohort study focusing on the initial glucose level as a prognostic factor for patients with first acute myocardial infarction. All adult patients (older than 18 years) admitted to the ED in a tertiary teaching hospital in Taiwan from January 1, 2001, to December 31, 2003, with a recorded diagnosis of acute myocardial infarction were eligible. We assessed the subject eligibility by admission diagnoses of acute myocardial infarction. We excluded the cases that did not fulfill the criteria of acute myocardial infarction diagnosis. If the patient was excluded, the medical record was rechecked by 2 investigators (S-JC, Y-SS). The protocol was approved by institutional review board in the hospital.

The medical records of all patients with acute myocardial infarction were retrieved according to International Classification of Diseases, Ninth Revision codes 410.xx by admission diagnosis. Specially trained nurses and a medical record technologist abstracted detailed information from the medical records of each patient, including documentation of the clinical history of cardiac risk factors, demographic characteristics, and particularly initial glucose levels at the ED. Information was collected on hospitalization, including morbidity (infections such as pneumonia and urinary tract infections), rehospitalization within 1 year because of cardiovascular events (recurrent angina or cardiovascular accident) for long-term outcome, site of myocardial infarction, congestive heart failure, cardiogenic shock, occurrence of ventricular defibrillation/ventricular tachycardia, reperfusion therapies including percutaneous coronary angioplasty, percutaneous coronary intervention with stent, and coronary artery bypass grafting. Two trained nurses and technologists reviewed all the extracted data in the medical records. If the patients did not meet the inclusion criteria or there were differences between them, or if there was a question especially about the adverse events for data extracting, the exclusion was rechecked or confirmed by at least 1 of the study physicians. The follow-up of long-term outcome was assessed by primary review of subsequent hospital records, national death registrations, and telephone follow-up interview by trained nurses.

Acute myocardial infarction was defined by the European Society of Cardiology/American College of Cardiology criteria: typical increase and gradual decrease (troponin) or more rapid increase and decrease creatine kinase-MB (CK-MB) of biochemical markers of myocardial necrosis and ischemic symptoms (mainly constrictive chest pain lasting more than 30 minutes) or ECG changes indicative of ischemia (ST-segment elevation or depression on at least 2 derivations). ${ }^{15}$ Patients were excluded if they did not meet the European Society of Cardiology/American College of Cardiology criteria or they had one of the following 4 criteria: (1) a previous episode of acute myocardial infarction; (2) acute myocardial infarction diagnosed at another hospital and referred for further treatment; (3) duration of acute myocardial infarction more than 24 hours; and (4) a history of percutaneous coronary intervention or coronary artery bypass grafting.

\section{Methods of Measurement}

Data were collected by a retrospective review of medical records. Venous blood samples were routinely drawn in the ED. Various laboratory values were collected from medical records. The patients were classified into 3 groups according to their glucose levels, as indicated by the initial tests in the ED. We defined initial glucose levels as normal $(<140 \mathrm{mg} / \mathrm{dL})$, intermediate (140 to $200 \mathrm{mg} / \mathrm{dL}$ ), and high ( $\geq 200 \mathrm{mg} / \mathrm{dL}$ ). The cutoffs of glucose values in our studies were based on diagnostic criteria for impaired glucose intolerance and diabetes mellitus during an oral glucose tolerance test. ${ }^{16}$ Using the 3 levels, we could also determine whether the patients had impaired glucose intolerance or diabetes mellitus later. We used 3 levels for predicting both short-term and long-term outcomes.

Diabetes mellitus was defined when the patient reported a history of diabetes or was receiving treatment with an oral hypoglycemic agent or insulin. Hypertension was defined as a 
systolic/diastolic blood pressure of greater than or equal to 140/ $90 \mathrm{~mm} \mathrm{Hg}$ or treatment with an antihypertensive agent.

Smoking status was recorded as either "never smoked" or "smoker" (current or former smoker). Hyperlipidemia was defined as having a reported history of increased fasting concentrations of total cholesterol or triglycerides or undergoing treatment with a hypolipidemic agent. Patients were defined as having a history of coronary artery disease if they had a history of acute myocardial infarction, coronary artery bypass grafting, or percutaneous coronary intervention. Intervention therapy, including primary percutaneous coronary intervention or coronary artery bypass grafting, was performed in suitable situations. The infarct site of acute myocardial infarction was assessed by at least 2 adjacent $S T$ elevations in precordial leads V2-4 (anterior wall) or leads II, III, and aVF (inferior wall). Lateral wall myocardial infarction was assessed by leads I, aVL, V5, or V6. Non-ST-elevation myocardial infarction was identified by no increase of the ST segment despite combined myocardial ischemic pain and increased cardiac enzyme activity.

\section{Outcome Measures}

We defined 1-month inhospital mortality as a primary endpoint and "composite short-term event" as a secondary endpoint. The composite short-term event included the occurrence of at least 1 of the following events in the hospital: 1-month mortality; cardiogenic shock, defined by a systolic blood pressure of less than $90 \mathrm{~mm} \mathrm{Hg}$ and a prolonged hypointensive episode with poor peripheral perfusion and not responsive to fluids or requiring institution of inotropic infusions to maintain blood pressure; ventricular fibrillation/ ventricular tachycardia; congestive heart failure, defined by dyspnea with concurrent interstitial edema on chest radiograph; and hospital-acquired infection.

We defined the following as "long-term outcomes": 1-year mortality: the patient died of any cause within 1 year of the onset of acute myocardial infarction and 1-year rehospitalization because of a major cardiovascular event, including recurrent angina or stroke.

\section{Primary Data Analysis}

All continuous variables are presented as means \pm SD. The baseline characteristics of the groups categorized by glucose levels were compared using the $\chi^{2}$ test or Fisher's exact test for independent variables in categorical variables. In analyzing the short-term outcomes, logistic regression was used because there was no exact time of event occurrence. Proportional hazards regression was used for analyzing the long-term prognosis because the exact date of event occurrence could be traced. To assess the adjusted effects of different glucose levels, we first selected the variables either having statistic $P<.05$ in the initial univariate results or clinically important factors for the events into multivariate analysis by logistic regression or proportional hazards regression. If a parameter was statistically significant $(P<.05)$, it was selected into multivariate models for predicting the prognoses of first acute myocardial infarction. To identify subsets of the risk factors that were most associated with the prognosis of first acute myocardial infarction, the stepwise regression was used for the best subset selection procedure of variables selection, with criteria of entry at significance level .2 and removal at significance level .2.

Kaplan-Meier survival analysis for long-term prognosis was used to estimate survival distribution according to the 3 groups of initial glucose levels in the ED. The significance of differences between the curves was tested using the log-rank statistic. Area under the receiver operating characteristic (ROC) curve was used to define the cutoff point of initial glucose level for predicting prognosis. SAS was used to perform the above analyses (version 9.1; SAS Institute, Inc., Cary, NC).

\section{RESULTS}

\section{Characteristics of the Study Subjects}

During the study period, 292 patients were admitted to the ED with acute myocardial infarction, but only 198 were eligible to participate in the study. Of the 292 patients, 94 patients were excluded because of previous percutaneous coronary intervention or coronary artery bypass grafting (13 patients), acute myocardial infarction more than 24 hours' duration (12 patients), or referral from another hospital (44 patients) or because it was not their first acute myocardial infarction ( 25 patients).

Of the 198 study patients (159 men and 39 women; mean age $63 \pm 14$ years), 94 had an initial glucose level of less than $140 \mathrm{mg} / \mathrm{dL}$ (normal group), $53 \mathrm{had}$ an initial glucose level of 140 to $200 \mathrm{mg} / \mathrm{dL}$ (intermediate group), and 51 had an initial glucose level of greater than or equal to $200 \mathrm{mg} / \mathrm{dL}$ (high group). Sixty-three patients (32\%) had a previous diagnosis of diabetes. Only $30(15 \%)$ patients were treated without any thrombolytic therapy. Patients with short- or long-term adverse outcomes after acute myocardial infarction were significantly older, had higher initial glucose levels, had higher peak CK activity, were more likely to be female or diabetic, and were less likely to have received reperfusion therapy than patients without adverse outcomes. There were no significant differences in hyperlipidemia, hypertension, and initial CK levels in the ED between patients with short- or long-term adverse outcomes and those without. For the infarct sites in acute myocardial infarction, anterolateral wall myocardial infarction was the most common site but was not statistically significant in patients with either short- or long-term adverse outcomes, or those without (Table 1).

There was an increasingly higher risk for short-term adverse outcome with increasing initial glucose levels compared with that of the normal group in the intermediate group and high group (1 versus 5.07 versus 6.47 in odds ratio) (Table 1 ).

\section{Main Results}

More than $40 \%$ of patients in the intermediate or high group had at least 1 short-term adverse outcome event; $18 \%$ in the normal group. Patients with high initial glucose levels had a significantly higher rate of cardiogenic shock, inhospital 
Table 1. Basic characteristics among study population, specified by composite short-term and long-term events.

\begin{tabular}{|c|c|c|c|c|c|c|c|c|c|c|c|c|}
\hline \multirow{3}{*}{$\begin{array}{l}\text { Characteristics } \\
\text { Male, \% }\end{array}$} & \multicolumn{6}{|c|}{ Short-Term Event } & \multicolumn{6}{|c|}{ Long-Term Event } \\
\hline & \multicolumn{2}{|c|}{ Present $(n=75)$} & \multicolumn{2}{|c|}{ Absent $(n=123)$} & \multirow{2}{*}{$\begin{array}{r}\begin{array}{r}\text { Odds } \\
\text { Ratio }\end{array} \\
0.57\end{array}$} & \multirow{2}{*}{$\begin{array}{c}\mathbf{9 5} \% \mathbf{~ C l} \\
0.28,1.16\end{array}$} & \multicolumn{2}{|c|}{ Present $(n=78)$} & \multicolumn{2}{|c|}{ Absent $(n=120)$} & \multirow{2}{*}{$\begin{array}{r}\begin{array}{c}\text { Hazard } \\
\text { Ratio }\end{array} \\
0.61\end{array}$} & \multirow{2}{*}{$\begin{array}{c}\mathbf{9 5 \%} \mathbf{~ C l} \\
0.37,1.02\end{array}$} \\
\hline & 56 & 75 & 103 & 84 & & & 58 & 74 & 101 & 84 & & \\
\hline Diabetes mellitus, \% & 33 & 44 & 30 & 24 & 2.44 & $1.32,4.50$ & 29 & 37 & 34 & 28 & 1.41 & $0.89,2.24$ \\
\hline Smokers, \% & 35 & 47 & 75 & 61 & 0.56 & $0.31,1.00$ & 37 & 47 & 73 & 61 & 0.66 & $0.42,1.02$ \\
\hline Hypertension, \% & 47 & 65 & 62 & 50 & 1.65 & $0.92,2.97$ & 49 & 63 & 60 & 50 & 1.52 & $0.96,2.41$ \\
\hline Hyperlipidemia, \% & 19 & 25 & 31 & 25 & 1.01 & $0.52,1.95$ & 19 & 24 & 31 & 26 & 0.92 & $0.55,1.54$ \\
\hline \multicolumn{13}{|l|}{ Initial ED glucose level, \% } \\
\hline Normal & 17 & 23 & 77 & 63 & 1 & & 26 & 33 & 68 & 57 & 1 & \\
\hline Intermediate & 28 & 37 & 25 & 20 & 5.07 & $2.39,10.8$ & 22 & 28 & 31 & 26 & 1.66 & $0.94,2.92$ \\
\hline High & 30 & 40 & 21 & 17 & 6.47 & $3.01,13.9$ & 30 & 39 & 21 & 17 & 2.90 & $1.71,4.91$ \\
\hline \multicolumn{13}{|l|}{ Infarct site, \% } \\
\hline Non-ST-elevation MI & 13 & 17 & 33 & 27 & 1 & & 13 & 17 & 33 & 28 & 1 & \\
\hline Anterolateral & 39 & 52 & 56 & 46 & 1.77 & $0.83,3.78$ & 37 & 47 & 58 & 48 & 1.54 & $0.82,2.90$ \\
\hline Inferoposterior & 23 & 31 & 34 & 27 & 1.72 & $0.75,3.94$ & 28 & 36 & 29 & 24 & 1.90 & $0.98,3.66$ \\
\hline Congestive heart failure, $\%$ & - & - & - & - & - & - & 18 & 23 & 17 & 14 & 1.68 & $0.99,2.85$ \\
\hline Cardiogenic shock, \% & - & - & - & - & - & - & 22 & 28 & 10 & 8 & 3.37 & $2.05,5.53$ \\
\hline $\begin{array}{l}\text { Receiving reperfusion } \\
\text { therapy, \% }\end{array}$ & 58 & 77 & 110 & 89 & 0.40 & $0.18,0.89$ & 58 & 77 & 110 & 89 & 0.59 & $0.34,1.04$ \\
\hline Age, y, SD & 69.1 & 13.4 & 59.5 & 13.5 & 9.6* & $5.7,13.5$ & 65.9 & 13.7 & 61.3 & 14.3 & $4.6 *$ & $0.6,8.7$ \\
\hline ED glucose, mg/dL, SD & 216.1 & 118 & 157.1 & 73.2 & 59.0* & $31.9,86.0$ & 201.9 & 111 & 165.5 & 84.5 & $36.4 *$ & $8.7,64.1$ \\
\hline Total cholesterol, mg/dL, SD & 179.3 & 79 & 192.4 & 49.3 & $-13.1 *$ & $-34.6,8.3$ & 192.3 & 75.9 & 185.7 & 49.5 & 6.6* & $-14.2,27.4$ \\
\hline HDL cholesterol, mg/dL, SD & 39.1 & 13.9 & 39.8 & 15.9 & $-0.7^{*}$ & $-7.2,5.6$ & 41.0 & 15.7 & 37.1 & 14.3 & $-3.9 *$ & $-10.0,2.2$ \\
\hline $\mathrm{CK}$ in $\mathrm{ED}, \mathrm{U} / \mathrm{L}, \mathrm{SD}$ & 711 & 1,171 & 603 & 994 & 108* & $-200,415$ & 803.5 & 1230 & 539.9 & 929.5 & $263.6 *$ & $-39.8,567$ \\
\hline $\begin{array}{l}\text { Peak CK during } \\
\text { hospitalization, U/L, SD }\end{array}$ & 2,639 & 2,498 & 1,968 & 1,842 & $671 *$ & $40,1,303$ & 2,474 & 2171 & 2,046 & 2,091 & 428* & $-200,1,055$ \\
\hline
\end{tabular}

$H D L$, High density lipoprotein; MI, myocardial infarction.

*Difference, not odds ratio or hazard ratio.

infection, ventricular fibrillation/ventricular tachycardia, and congestive heart failure in the intermediate group and high group compared with the normal group. About the primary endpoint, 30-day mortality rate was higher in the intermediate and high groups than in the normal group (3\% versus $13 \%$ versus $14 \%$ ) but was not significantly different (Table 2).

Higher initial glucose levels in the ED were associated with a higher rate of 1-year long-term prognosis. There was an increasingly higher risk for long-term outcome with increasing initial glucose levels compared with that of the normal group ( 1 versus 1.66 versus 2.90 in odds ratio) (Table 1 ). Of the total number of patients, there was $10 \%$ mortality, $28 \%$

rehospitalization for recurrent angina, and 2\% rehospitalization for stroke, and $29 \%$ had at least 1 of the events. Patients with high initial glucose levels had a significantly higher rate of 1-year mortality (3\% versus $15 \%$ versus $18 \%$ ), rehospitalization for recurrent angina (21\% versus $28 \%$ versus $41 \%)$, and 1 -year cardiovascular events ( $25 \%$ versus $28 \%$ versus $41 \%$ ) in the intermediate group and the high group compared with the normal group (Table 3).

The estimates of survival in long-term prognosis of patients at the 3 initial glucose levels in the ED were analyzed with the Kaplan-Meier survival curves, which showed a significant increase in the rate of long-term adverse outcomes with increasing initial glucose levels (log-rank test, $P=.002$ ) (Figure).

The adjusted odds ratio for short-term prognosis increased progressively with higher levels of initial glucose compared with that of the normal level (odds ratio 3.87 in the intermediate group; 5.16 in the high group). A Cox model showed a significant positive association between long-term prognosis and the high group (hazard ratio 3.08 ; $95 \%$ CI 1.59 to 5.98 ) after adjusting for sex, age, diabetic status, reperfusion therapy, and infarct site. There was also a positive association with the infarct site of the inferoposterior wall (hazard ratio 2.09; 95\% CI 1.05 to 5.98) after multivariate analyses.

By using variables of initial glucose level, sex, age, diabetic status, reperfusion therapy, and infarct subtype, the stepwise logistic regression model selection showed that the major determinants of short-term prognosis in the total group were initial glucose levels of the intermediate or high group and age. Stepwise Cox regression model selection indicated that only initial glucose levels of the high group, age, and inferoposterior wall myocardial infarction subtype were major determinants of long-term prognosis (Table 4).

The mean initial glucose level in the ED was 262.6 \pm 125.5 $\mathrm{mg} / \mathrm{dL}$ in diabetic patients and $142.9 \pm 47.4 \mathrm{mg} / \mathrm{dL}$ in nondiabetic patients. There was no significant interaction between initial glucose level in the ED and diabetic status for short-term (intermediate level $\times$ diabetes, $P=.575$; high level $\times$ diabetes, $P=.839$ ) or long-term events (intermediate level $\times$ diabetes, $P=$.986; high level $\times$ diabetes, $P=.452$ ). Older and female patients were predominant among acute myocardial infarction patients with diabetes. Patients with a higher initial glucose level had a higher ratio of diabetes compared with those in the normal group (16\% versus $24 \%$ versus $60 \%$ ). The 
Table 2. One-month short-term prognoses in the study population, specified by initial glucose levels.*

\begin{tabular}{|c|c|c|c|c|}
\hline Characteristics & $\begin{array}{l}\text { Total, } \\
\mathrm{N}=198\end{array}$ & $\begin{array}{c}\text { Normal Group, } \\
\quad N=94\end{array}$ & $\begin{array}{l}\text { Intermediate Group, } \\
\qquad N=53\end{array}$ & $\begin{array}{l}\text { High Group, } \\
\quad \mathrm{N}=51\end{array}$ \\
\hline \multicolumn{5}{|l|}{ Cardiogenic shock } \\
\hline Case & $32(16)$ & $9(10)$ & $10(19)$ & $13(26)$ \\
\hline Odds ratio $(95 \% \mathrm{Cl})$ & & 1 & $2.20(0.83-5.81)$ & $3.23(1.27-8.21)$ \\
\hline \multicolumn{5}{|c|}{ Infection: including urinary tract infection, pneumonia } \\
\hline Case & $28(14)$ & $7(7)$ & $7(13)$ & $14(27)$ \\
\hline Odds ratio $(95 \% \mathrm{Cl})$ & & 1 & $1.89(0.63-5.72)$ & $4.70(1.76-12.60)$ \\
\hline \multicolumn{5}{|c|}{ Ventricular fibrillation/tachycardia } \\
\hline Case & $15(8)$ & $3(3)$ & $3(6)$ & $9(18)$ \\
\hline Odds ratio $(95 \% \mathrm{Cl})$ & & 1 & $1.82(0.35-9.36)$ & $6.50(1.67-25.25)$ \\
\hline \multicolumn{5}{|c|}{ Congestive heart failure } \\
\hline Case & $35(18)$ & $9(10)$ & $12(23)$ & $14(27)$ \\
\hline Odds ratio $(95 \% \mathrm{Cl})$ & & 1 & $2.76(1.08-7.09)$ & $3.57(1.42-8.99)$ \\
\hline \multicolumn{5}{|c|}{ 30-Day death during hospitalization } \\
\hline Case & $17(9)$ & $3(3)$ & $7(13)$ & $7(14)$ \\
\hline Odds ratio $(95 \% \mathrm{Cl})$ & & 1 & $3.42(0.95-12.30)$ & $3.58(1.00-12.87)$ \\
\hline \multicolumn{5}{|c|}{ Numbers of above events } \\
\hline 0 & $123(62)$ & $77(82)$ & $25(47)$ & $21(41)$ \\
\hline 1 & $42(21)$ & $10(11)$ & $18(34)$ & $14(27)$ \\
\hline 2 & $15(8)$ & $1(1)$ & $8(15)$ & $6(12)$ \\
\hline 3 & $14(7)$ & $5(5)$ & $2(4)$ & $7(14)$ \\
\hline 4 & $4(2)$ & $1(1)$ & 0 & $3(6)$ \\
\hline
\end{tabular}

Table 3. One-year long-term prognosis in the study population, specified by glucose levels.*

\begin{tabular}{|c|c|c|c|c|}
\hline Characteristics & $\begin{array}{l}\text { Total, } \\
\mathrm{N}=198\end{array}$ & $\begin{array}{l}\text { Normal Group, } \\
\quad \mathrm{N}=94\end{array}$ & $\begin{array}{l}\text { Intermediate Group, } \\
\qquad \mathrm{N}=\mathbf{5 3}\end{array}$ & $\begin{array}{l}\text { High Group, } \\
\quad \mathrm{N}=51\end{array}$ \\
\hline \multicolumn{5}{|l|}{ One-year mortality } \\
\hline Case & $20(10)$ & $3(3)$ & $8(15)$ & $9(18)$ \\
\hline Hazard ratio $(95 \% \mathrm{Cl})$ & & 1 & $4.94(1.31-18.63)$ & $6.40(1.73-23.66)$ \\
\hline \multicolumn{5}{|c|}{ Recurrent angina rehospitalization } \\
\hline Case & $56(28)$ & $20(21)$ & $15(28)$ & $21(41)$ \\
\hline Hazard ratio (95\% Cl) & & 1 & $1.49(0.76-2.91)$ & $2.78(1.51-5.13)$ \\
\hline \multicolumn{5}{|c|}{$\begin{array}{l}\text { Cardiovascular events: recurrent angina or } \\
\text { stroke rehospitalization }\end{array}$} \\
\hline Case & $59(30)$ & $23(25)$ & $15(28)$ & $21(41)$ \\
\hline Hazard ratio (95\% Cl) & & 1 & $1.30(0.68-2.49)$ & $2.42(1.34-4.38)$ \\
\hline \multicolumn{5}{|c|}{ Numbers of above events } \\
\hline 0 & $139(70)$ & $68(72)$ & $31(58)$ & 21 (41) \\
\hline 1 & $56(28)$ & $23(25)$ & $21(40)$ & $30(59)$ \\
\hline 2 & $3(2)$ & $3(3)$ & $1(2)$ & 0 \\
\hline
\end{tabular}

mortality rate showed no significant difference both in shortterm and long-term prognoses. However, there was a significantly higher risk for short-term event (odds ratio 2.25; 95\% CI 1.25 to 4.04 ) among diabetic patients than those without diabetes. The long-term event showed no statistically significant difference between diabetic and nondiabetic patients (Table 5).

Higher initial glucose levels were associated with an increased rate of short-term events and long-term events in the intermediate group and high group compared with the normal group in nondiabetic patients. However, this trend was not observed significantly in statistical analysis in diabetic patients (data not shown).
The area under the ROC curve for initial glucose levels to predict composite short- and long-term events in first acute myocardial infarction patients was 0.70 and 0.63 , respectively. The cutoff point of initial glucose level for predicting short-term events was $180 \mathrm{mg} / \mathrm{dL}$ (sensitivity 50.7\%; specificity 75.6\%).

\section{LIMITATIONS}

First, the study design was a nonrandomized observational study. Our study population was also relatively small, with a male predominance. However, it reflected the ED population in a tertiary teaching hospital in that it included all consecutive acute myocardial infarction patients. Second, we did not have detailed medication histories of the study group 


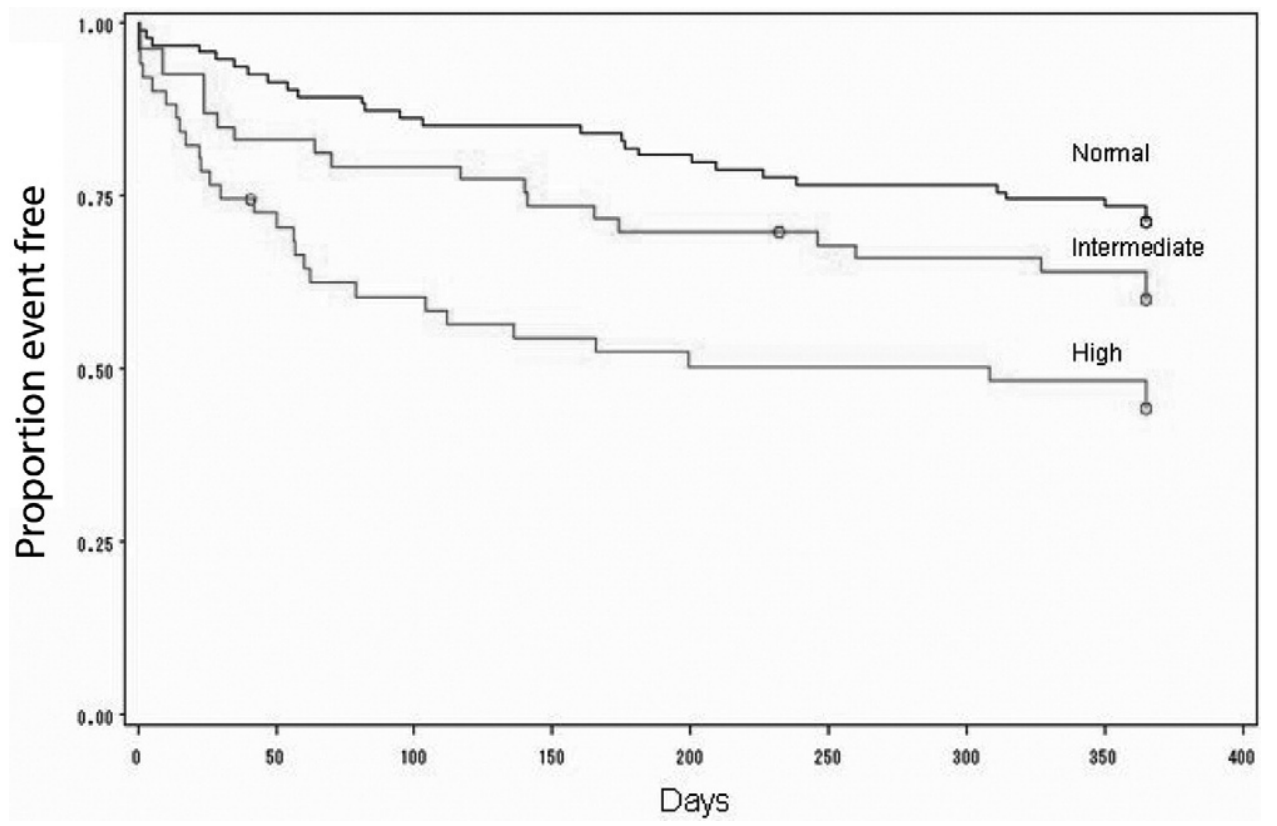

Figure. The Kaplan-Meier cumulative survival curves for patients during the follow-up period of 1 year, stratified by the 3 initial glucose levels (log-rank test, $P=.0002$ ).

Table 4. Multivariate analyses and model selection of predictors of composite short-term and long-term events in patients with first acute myocardial infarction.

\begin{tabular}{|c|c|c|c|c|}
\hline \multirow[b]{2}{*}{ Characteristics } & \multicolumn{2}{|c|}{ OR $(95 \% \mathrm{CI})$} & \multicolumn{2}{|c|}{ HR (95\% Cl) } \\
\hline & $\begin{array}{l}\text { Short-Term Prognosis } \\
\text { (Model I)* }\end{array}$ & $\begin{array}{l}\text { Short-Term Prognosis } \\
\text { (Model II)* }\end{array}$ & $\begin{array}{l}\text { Long-Term Prognosis } \\
\text { (Model III)* }\end{array}$ & $\begin{array}{l}\text { Long-Term Prognosis } \\
\text { (Model IV)* }\end{array}$ \\
\hline Intermediate/normal level & $3.87(1.71-8.78)$ & $4.10(1.87-9.03)$ & $1.45(0.80-2.61)$ & \\
\hline High/normal level & $5.16(1.97-13.51)$ & $6.08(2.74-13.49)$ & $3.08(1.59-5.98)$ & $2.13(1.34-3.40)$ \\
\hline Sex, male/female & $1.11(0.47-2.60)$ & & $0.80(0.46-1.41)$ & \\
\hline Age, y & 1.05 (1.02-1.08) & 1.05 (1.02-1.08) & $1.02(0.99-1.04)$ & $1.02(1.00-1.04)$ \\
\hline Diabetic status & $1.11(0.48-2.53)$ & & $0.68(0.39-1.25)$ & \\
\hline Receiving reperfusion therapy, yes/no & $0.56(0.22-1.44)$ & & $0.64(0.35-1.17)$ & $0.62(0.34-1.12)$ \\
\hline Anterolateral wall MI/non-ST elevation MI & $2.33(0.95-5.74)$ & & $1.52(0.79-2.93)$ & $1.61(0.84-3.07)$ \\
\hline Inferoposterior wall MI/non-ST elevation MI & $2.46(0.90-6.73)$ & & $2.09(1.05-5.98)$ & $2.29(1.16-4.53)$ \\
\hline
\end{tabular}

or clinical features such as high-degree atrioventricular block and renal function of the study group, which may have influenced the outcomes of acute myocardial infarction. Third, we decided to exclude patients with other than first acute myocardial infarction and those with acute myocardial infarction of more than 24 hours' duration. Therefore, the validity of the study may be limited to first acute myocardial infarction without previous coronary artery disease in ED populations. Finally, we could not determine the incidence of diabetes mellitus when patients had no history of diabetes. However, an admission diagnosis of diabetes is highly specific, and most patients will declare that they have a history of diabetes. Further prospective studies are required to clarify the effect of when to start intensive treatment and how to monitor acute hyperglycemia.

\section{DISCUSSION}

We clearly demonstrated the positive relationship between initial glucose level and the short- and long-term prognoses in first acute myocardial infarction. Our study showed that patients with first acute myocardial infarction and high initial glucose levels in the ED represent a high-risk population. The hyperglycemia conferred a risk that was independent of sex, age, diabetic status, infarct site, and reperfusion therapy, suggesting that glucose status itself may be a key marker of adverse cardiac outcome after acute myocardial infarction. The highest initial 
Table 5. Short-term and long-term prognosis in diabetic and nondiabetic patients with first acute myocardial infarction.

\begin{tabular}{|c|c|c|c|c|}
\hline \multirow[b]{2}{*}{ Characteristics } & \multicolumn{4}{|c|}{ Diabetes Mellitus } \\
\hline & Present $(n=63)$ & Absent $(n=135)$ & Odds (Hazard) Ratio & $95 \% \mathrm{Cl}$ \\
\hline \multicolumn{5}{|l|}{ Demographic characteristics } \\
\hline Age, y, SD & $66.3 \pm 11.6$ & $61.5 \pm 15$ & $P=0.011$ & \\
\hline Male, \% & $44(70)$ & $115(85)$ & 0.40 & $(0.20-0.83)$ \\
\hline \multicolumn{5}{|l|}{ Initial ED glucose level, \% } \\
\hline Normal & $10(16)$ & $84(62)$ & 1.00 & \\
\hline Intermediate & $15(24)$ & $38(28)$ & 3.32 & $(1.37-8.05)$ \\
\hline High & $38(60)$ & $13(10)$ & 24.55 & $(9.89-60.95)$ \\
\hline \multicolumn{5}{|l|}{ Short-term prognosis, \% } \\
\hline Cardiogenic shock & $10(16)$ & $22(16)$ & 0.97 & $(0.43-2.19)$ \\
\hline Infection, including urinary tract infection, pneumonia & $14(22)$ & $14(10)$ & 2.47 & $(1.10-5.56)$ \\
\hline Ventricular fibrillation/tachycardia & $6(10)$ & $9(7)$ & 1.47 & $(0.50-4.34)$ \\
\hline Congestive heart failure & $18(29)$ & $17(13)$ & 2.78 & $(1.32-5.85)$ \\
\hline 30-Day death during hospitalization & $7(11)$ & $11(8)$ & 1.41 & $(0.52-3.83)$ \\
\hline Short-term event & $33(52)$ & $42(31)$ & 2.25 & $(1.25-4.04)$ \\
\hline \multicolumn{5}{|l|}{ Long-term prognosis, \% } \\
\hline One-year mortality & $8(13)$ & $12(9)$ & 1.53 & $(0.62-3.74)$ \\
\hline Cardiovascular events: recurrent angina or stroke rehospitalization & $21(33)$ & $38(28)$ & 1.34 & $(0.79-2.28)$ \\
\hline Long-term event & $29(46)$ & $49(36)$ & 1.41 & $(0.89-2.24)$ \\
\hline
\end{tabular}

glucose level was an independent predictive factor for both short- and long-term prognoses after first acute myocardial infarction.

The duration from symptom onset to obtaining glucose level was less than 12 hours in our study population (80\%). The short duration of the available data might represent the peak glucose level that reflects the massive stress hormone effect. The mechanisms of hyperglycemia associated with short- and longterm prognoses of acute myocardial infarction were different. For short-term outcome, acute stress-related events were pointed. The mechanism underlying short-term adverse cardiovascular events may involve the effects of stress hormones or the extent of the myocardial infarct itself. ${ }^{17-19}$ The more severe the stress, the greater the surge of serum catecholamines secreted and the stronger the glucose response that is stimulated. Plasma catecholamines have been reported to be associated with a higher risk of cardiogenic shock after acute myocardial infarction. ${ }^{20}$ In turn, hyperglycemia may be a consequence of a larger infarct size and may just reflect the extent of severe injury or cardiac damage.

For long-term prognosis, hyperglycemia may augment thrombus formation and reduce collateral circulation, resulting in a larger infarct size. ${ }^{21}$ Why is a higher initial glucose level independently associated with long-term prognosis after first acute myocardial infarction? First, $75 \%$ of patients with high initial glucose levels were diabetic in our study population. High initial glucose status may reflect potentially uncontrolled increased blood glucose, which can increase the risk of macroand microvascular morbidity and influence the long-term adverse cardiovascular events after acute myocardial infarction. Second, acute hyperglycemia in the nondiabetic population could represent individuals with undiagnosed diabetes or impaired glucose metabolism. ${ }^{22}$ The incidence of unrecognized diabetes was $65 \%$ in acute myocardial infarction patients with an initial glucose response that exceeded $180 \mathrm{mg} / \mathrm{dL}^{23}$

In a prospective study of acute myocardial infarction in both nondiabetic patients and patients with undiagnosed diabetes, increased glycosylation persisted for 4 to 8 weeks. ${ }^{24}$ If stress hyperglycemia does reflect an underlying glucose intolerance, this would correlate with a higher risk not only of short-term adverse cardiac outcomes but also of long-term cardiovascular events after acute myocardial infarction. Thus, it may be unnecessary to control stress hyperglycemia in acute myocardial infarction patients until the stress level decreases. If glucose intolerance or frank diabetes persists in the following 48 to 72 hours or up to 2 to 4 weeks, intensive metabolic treatment for hyperglycemia will be necessary to prevent long-term adverse cardiovascular events. ${ }^{25,26}$

Previous studies showed that in diabetic patients with acute myocardial infarction, the mortality increased with increasing initial glucose level. ${ }^{5,10,27}$ However, our studies showed that there was no significant difference among the 3 groups for the long-term event after the first acute myocardial infarction, whether the patient had diabetes or not. It may be because the sample size was too small to show any significant difference. Moreover, the adjusted hazard ratio of diabetes was not statistically significant.

Why was there no significant difference among the 3 initial glucose levels in the rate of short-term events in acute myocardial infarction patients with diabetes mellitus, which is not observed in acute myocardial infarction patients without diabetes mellitus? Initial glucose level in acute myocardial infarction patients was confounded by diabetes, and the glucose level did not reflect the real glucose response to stress of acute myocardial infarction, because of unreliable baseline glucose level. Hyperglycemia will not be a marker of short-term 
prognosis or inhospital death in acute myocardial infarction patients with diabetes mellitus because the hyperglycemia is not representative of glucose response to acute stress.

Iwakura et $\mathrm{al}^{21}$ stated that acute myocardial infarction patients with hyperglycemia have higher peak CK levels than those without. However, peak CK activity was not related to initial glucose levels in our study, which may be explained by the probability that peak CK levels were confounded by early reperfusion therapy and early death in our patients.

Furthermore, hyperglycemia appears to be related to an excessive stress hormone response, which is independent of the extent of infarction and not related to infarct size.

\section{In Retrospect}

The study might have been improved by collection of more confounding factors such as medication history and by intervention trial designs implemented with aggressive glucose levels.

In summary, the initial glucose level measured in the ED may be an important prognostic factor of short- and long-term cardiovascular outcomes after first acute myocardial infarction.

\section{Supervising editor: Judd E. Hollander, MD}

Author contributions: C-WH conceived the study, obtained funding, drafted the manuscript, and collected the data. HHC supervised the conduct of the study and conducted statistical analysis. WH-HS, S-JC Y-SS, and C-PY contributed to the design of the study and contributed to the data collection. KLC supervised the study procedure and provided statistical advice on study design, and all authors contributed substantially to its revision. K-LC takes responsibility for the paper as a whole.

Funding and support: This project was funded by a grant from the National Science Council in Taiwan.

Publication dates: Received for publication March 9, 2006. Revisions received June 16, 2006, and October 9, 2006. Accepted for publication October 23, 2006. Available online December 18, 2006.

Address for reprints: Kuo-Liong Chien, MD, PhD, Institute of Preventive Medicine, College of Public Health, National Taiwan University, No. 17, Hsu-Chou Road, Taipei, Taiwan 100; 886-2-3322-8017, fax 886-2-2392-0456; E-mail klchien@ ha.mc.ntu.edu.tw.

\section{REFERENCES}

1. Stubbs PJ, Laycock J, Aghband-Zadeh J, et al. Circulating stress hormone and insulin concentrations in acute coronary syndromes: identification of insulin resistance on admission. Clin Sci (Lond). 1999;96:589-595.

2. Capes SE, Hunt D, Malmberg K, et al. Stress hyperglycemia and increased risk of death after myocardial infarction in patients with and without diabetes: a systematic overview. Lancet. 2000;355: 773-778.

3. Malmberg K, Ryden L. Myocardial infarction in patients with diabetes mellitus. Eur Heart J. 1988;9:259-264.
4. Ishihara M, Inoue I, Kawagoe T, et al. Impact of acute hyperglycemia on left ventricular function after reperfusion therapy in patients with a first anterior wall acute myocardial infarction. Am Heart J. 2003;146:674-678.

5. Sewdarsen M, Vythilingum S, Jialal I, et al. Prognostic importance of admission plasma glucose in diabetic and non-diabetic patients with acute myocardial infarction. Q J Med. 1989;71:461466.

6. Hadjadj S, Coisne D, Mauco G, et al. Prognostic value of admission plasma glucose and $\mathrm{HbA}$ in acute myocardial infarction. Diabet Med. 2004;21:305-310.

7. Norhammar AM, Ryden L, Malmberg K. Admission plasma glucose. Independent risk factor for long-term prognosis after myocardial infarction even in nondiabetic patients. Diabetes Care. 1999;22:1827-1831.

8. Tenerz A, Nilsson G, Forberg R, et al. Basal glucometabolic status has an impact on long-term prognosis following an acute myocardial infarction in non-diabetic patients. J Intern Med. 2003; 254:494-503.

9. Timmer JR, van der Horst IC, Ottervanger JP, et al. Prognostic value of admission glucose in non-diabetic patients with myocardial infarction. Am Heart J. 2004;148:399-404.

10. Suleiman $M$, Hammerman $H$, Boulos $M$, et al. Fasting glucose is an important independent risk factor for 30-day mortality in patients with acute myocardial infarction: a prospective study. Circulation. 2005;111:754-760.

11. Wahab NN, Cowden EA, Pearce NJ, et al. Is blood glucose an independent predictor of mortality in acute myocardial infarction in the thrombolytic era? J Am Coll Cardiol. 2002;40:1748-1754.

12. Wong VW, Ross DL, Park K, et al. Hyperglycemia: still an important predictor of adverse outcomes following AMI in the reperfusion era. Diabetes Res Clin Pract. 2004;64:85-91.

13. Anand SS, Xie CC, Mehta S, et al. Differences in the management and prognosis of women and men who suffer from acute coronary syndromes. J Am Coll Cardiol. 2005;46:18451851.

14. Bartnik M, Malmberg K, Norhammar A, et al. Newly detected abnormal glucose tolerance: an important predictor of long-term outcome after myocardial infarction. Eur Heart J. 2004;25:19901997.

15. The Joint European Society of Cardiology/American College of Cardiology Committee. Myocardial infarction redefined: a consensus document of the Joint European Society of Cardiology/American College of Cardiology Committee for the Redefinition of Myocardial Infarction. Eur Heart J. 2000;21:15021513.

16. Qiao Q, Pyorala K, Pyorala M, et al. Two-hour glucose is a better risk predictor for incident coronary heart disease and cardiovascular mortality than fasting glucose. Eur Heart J. 2002; 23:1267-1275.

17. Bain RJ, Fox JP, Jagger J, et al. Serum cortisol levels predict infarct size and patient mortality. Int J Cardiol. 1992;37:145-150.

18. Vetter NJ, Strange RC, Adams W, et al. Initial metabolic and hormonal response to acute myocardial infarction. Lancet. 1974; 1:284-288.

19. Oswald GA, Smith CC, Betteridge DJ, et al. Determinants and importance of stress hyperglycaemia in non-diabetic patients with myocardial infarction. BMJ (Clin Res Ed). 1986;293:917-922.

20. Bain RJ, Fox JP, Jagger J, et al. Serum cortisol levels predict infarct size and patient mortality. Int J Cardiol. 1992;37:145-150.

21. Iwakura $\mathrm{K}$, Ito $\mathrm{H}$, Ikushima $\mathrm{M}$, et al. Association between hyperglycemia and the no-reflow phenomenon in patients with acute myocardial infarction. J Am Coll Cardiol. 2003;41:1-7.

22. Madsen JK, Haunsoe S, Helquist S, et al. Prevalence of hyperglycaemia and undiagnosed diabetes mellitus in patients 
with acute myocardial infarction. Acta Med Scand. 1986;220:329-332.

23. Husband DJ, Alberti KG, Julian DG. “Stress” hyperglycaemia during acute myocardial infarction: an indicator of pre-existing diabetes? Lancet. 1983;2:179-181.

24. Chandalia HB, Gokani AH. Stress hyperglycaemia. Lancet. 1984; 2:811-812.

25. Malmberg K, Ryden L, Efendic S, et al. Randomized trial of insulin-glucose infusion followed by subcutaneous insulin treatment in diabetic patients with acute myocardial infarction
(DIGAMI study): effects on mortality at 1 year. J Am Coll Cardiol. 1995;26:57-65.

26. Malmberg K. Prospective randomised study of intensive insulin treatment on long term survival after acute myocardial infarction in patients with diabetes mellitus: DIGAMI (Diabetes Mellitus, Insulin Glucose Infusion in Acute Myocardial Infarction) Study Group. BMJ. 1997;314:1512-1515.

27. Rytter L, Troelsen S, Beck-Nielsen H. Prevalence and mortality of acute myocardial infarction in patients with diabetes. Diabetes Care. 1985;8:230-234.

You can save your online searches and get the results by e-mail.

Visit www.annemergmed.com today to see what else is new online! 